\title{
Nonextensive statistical mechanics, super- statistics and beyond: theory and applications in astrophysical and other complex systems
}

\author{
C. Beck ${ }^{1}$, G. Benedek ${ }^{2,3}$, G. Livadiotis ${ }^{4}$, A. Rapisarda ${ }^{5,6, a}$, U. Tirnakli ${ }^{7}$, \\ and C. Tsallis $8,9,10$ \\ ${ }^{1}$ School of Mathematical Sciences, Queen Mary University of London, London, UK \\ ${ }^{2}$ Department of Materials Science, Universitá di Milano Bicocca, Bicocca, Italy \\ ${ }^{3}$ Donostia International Physics Center, San Sebastian, Spain \\ ${ }^{4}$ Southwest Research Institute, San Antonio, TX, USA \\ ${ }^{5}$ Dipartimento di Fisica e Astronomia "Ettore Majorana" and Istituto Nazionale \\ di Fisica Nucleare, Universitá di Catania, Catania, Italy \\ ${ }^{6}$ Complexity Science Hub Vienna, Vienna, Austria \\ ${ }^{7}$ Department of Physics, Faculty of Science, Ege University, 35100 Izmir, Turkey \\ ${ }^{8}$ Centro Brasileiro de Pesquisas Fisicas and National Institute of Science and \\ Technology for Complex Systems, Rio de Janeiro, Brazil \\ ${ }^{9}$ Complexity Science Hub Vienna, Vienna, Austria \\ ${ }^{10}$ Santa Fe Institute, Santa Fe, NM, USA
}

Received 23 January 2020

Published online 12 March 2020

\begin{abstract}
A brief illustration is presented about the scientific motivation and contributions of this Special Issue.
\end{abstract}

\section{Introduction}

After more than 140 years of impressive success there is no reasonable doubt that the Boltzmann-Gibbs (BG) entropy is the correct one to be used for a wide and important class of physical systems. The latter ones are basically those systems whose (nonlinear) dynamics is strongly chaotic, such as classical systems with positive maximal Lyapunov exponent, which are mixing and ergodic. However, a plethora of other physical complex systems exists for which such simplifying dynamical hypotheses are violated. Typical examples are those for which the maximal Lyapunov exponent vanishes, leading to sub-exponential sensitivity to the initial conditions, which can of course occur in a variety of mathematical ways. Corresponding anomalies are found also in a variety of quantum systems.

In order to statistically describe the dynamics of such systems, various generalised forms of statistical mechanics have been proposed such as those using the nonadditive entropies $S_{q}$ (where $q$ is a real number which, for $q=1$, recovers the BG entropy), kappa distributions (also known as $q$-Gaussians, where kappa is simply related to $q$ ), superstatistical approaches, among various others. In the last decades, these new generalised statistical mechanical formalisms have found a large variety of very successful

a e-mail: andrea.rapisarda@ct.infn.it 
applications, many of them beyond the realm of physics. This special issue aims to cover some of the most recent analytical, experimental, observational and computational aspects and examples where these new extended formalisms have found fruitful applications. In this special volume we include some of those contributions which were presented at the XVI international course of the School of Complexity held in July 2-8 2019 at the Ettore Majorana Foundation and Center for Scientific Culture in Erice (Italy).

In particular, Metzler discusses superstatistics and non-Gaussian diffusion [1]; Zhang et al. [2] investigate a class of network diffusion equations with large powernonlinearity connected to $q$-statistics; Carati et al. [3] discuss via $q$-distributions a realistic ionic-crystal model and, within the FPU model, how the system reaches equilibrium; Rozynek and Wilk [4] study the level of nonextensivity of the quarkgluon system described by lattice QCD; Souza et al. investigate the ground entangled state of the one-dimensional spin-1/2 Ising ferromagnet at its transverse-field critical point [5]; Suyari et al. study the advantages of the $q$-logarithm representation over the $q$-exponential one [6]; Korbel et al. [7] discuss within the information geometry framework the scaling expansions of non-exponentially growing configuration spaces; Wedemann and Plastino [8] study a nonlinear Fokker Planck equation and its stationary-state solution, proving an $\mathrm{H}$-theorem obeyed by a free-energy functional that involves the generalized entropy $S_{q}$; Yoon uses the equivalence of kappa distributions and $q$-Gaussian distributions to focus on the non-equilibrium statistical mechanical applications to the formation of non-Maxwellian electron distribution in space [9]. Interesting applications of $q$-statistics for predicting ruptures and earthquakes are then discussed in the paper by Greco et al [10] and in the paper by Skordas et al. [11] respectively; Singh and Roy [12] discuss an internetwork synchronisation technique for complex dynamical networks of different kinds.

We hope that these studies will stimulate further discussions opening new interesting research directions. At the ending of the 19th century it was unnecessary to qualify Mechanics with expressions such as Newtonian Mechanics or Classical Mechanics, commonly used nowadays. Indeed, only one such theory existed. With the emergence in theoretical physics of Einstein's special and general relativities and of quantum mechanics, the situation drastically changed as we all know. The same happened with the (additive) Boltzmann-Gibbs entropy (or entropic functional): it was just called entropy during nearly one century and a half. The set of works included in the present special issue, with their direct connections to nonadditive entropic functionals, neatly illustrate the present need, in contemporary theoretical physics, to specifically qualify as $B G$ entropy and BG statistical mechanics, the concept and associated thermostatistical theory that emerged at the end of the 19th century and which is today taught and used world-wide in the courses of physics, chemistry, computer science, mathematics, and elsewhere.

Finally, we warmly thank all the contributors for sharing their studies, and express our gratitude to the editorial members of EPJST for inviting this special issue.

\section{References}

1. R. Metzler, Eur. Phys. J. Special Topics 229, 711 (2020)

2. X. Zhang, T. Senyo, H. Sakai, A. Ohara, Eur. Phys. J. Special Topics 229, 729 (2020)

3. A. Carati, L. Galgani, F. Gangemi, R. Gangemi, Eur. Phys. J. Special Topics 229, 743 (2020)

4. J. Rozynek, G. Wilk, Eur. Phys. J. Special Topics 229, 751 (2020)

5. A.M.C. Souza, P. Rapcan, C. Tsallis, Eur. Phys. J. Special Topics 229, 759 (2020)

6. H. Suyari, H. Matsuzoe, A.M. Scarfone, Eur. Phys. J. Special Topics 229, 773 (2020) 
7. J. Korbel, R. Hanel, S. Thurner, Eur. Phys. J. Special Topics 229, 787 (2020)

8. R.S. Wedemann, A.R. Plastino, Eur. Phys. J. Special Topics 229, 809 (2020)

9. P.H. Yoon, Eur. Phys. J. Special Topics 229, 819 (2020)

10. A. Greco, C. Tsallis, A. Rapisarda, A. Pluchino, G. Fichera, L. Contrafatto, Eur. Phys. J. Special Topics 229, 841 (2020)

11. E.S. Skordas, N.V. Sarlis, P.A. Varotsos, Eur. Phys. J. Special Topics 229, 851 (2020)

12. P.P. Singh, B.K. Roy, Eur. Phys. J. Special Topics 229, 861 (2020) 